\title{
Noticias del arpa de dos órdenes en la Nueva España y el México decimonónico ${ }^{1}$
}

\author{
Information about the Spanish Double Harp in \\ New Spain and Nineteenth-century Mexico
}

El arpa española de dos órdenes fue ampliamente utilizada como instrumento realizador del bajo continuo en la península ibérica durante el siglo XVII y hasta mediados del XVIII. Hallazgos documentales recientes permiten probar que este modelo de arpa también se integró en las prácticas musicales del Nuevo Mundo. El presente artículo se centra en el caso de la Nueva España, donde fuentes escritas revelan su uso en las capillas catedralicias de Puebla y Valladolid (Michoacán) durante el primer tercio del siglo XVIII. De igual forma, estos testimonios acreditan que el instrumento cromático fue construido en el virreinato de México y que en la época hubo un fuerte interés por contratar músicos que supieran tañerlo. Además se ofrecen noticias de su presencia en el ámbito popular-quizás de forma excepcional- durante el siglo XIX.

Palabras clave: arpa de dos órdenes, Nueva España, ámbito catedralicio, México.

The Spanish double harp was widely used as the instrument that played the basso continuo in the Iberian Peninsula during the seventeenth and until the mid-eighteenth centuries. Recently discovered documents substantiate that this type of harp was also part of music making in the New World. The present article focuses on the case of New Spain, where documentary sources reveal its use in the Puebla and Valladolid (Michoacan) cathedral chapels during the first third of the eighteenth century. Likewise, these testimonies demonstrate that the chromatic instrument was constructed in the Viceroyalty of Mexico and that during this period there was a strong interest in hiring musicians who knew how to play it. Information about its presence in the popular sphere-perhaps exceptionallyduring the nineteenth century is also provided.

Keywords: Spanish double harp, New Spain, cathedral chapels, Mexico.

A partir de la década de 1950, después de que en los años treinta Nicanor Zabaleta llamara la atención sobre la conservación de un arpa de dos órdenes ${ }^{2}$, el mismo arpista vasco dio inicio a los estudios sistemáticos sobre el tema, al que le siguieron trabajos de autores como Hans Joaquim Zingel, Santiago Kastner, Robert Stevenson, Rafael Pérez Arroyo, José López-Calo, Cristina Bordas, Egberto Bermúdez y Dagmar

${ }^{1}$ La información que aparece en este texto forma parte de la investigación doctoral que realizo en el Programa de Doctorado en Música de la Universidad Nacional Autónoma de México. Agradezco a Cristina Bordas y a Josemi Lorenzo Arribas la atenta lectura del primer borrador de este texto.

2 C. Bordas: "Reencuentro con el arpa de Juan López (Toledo ca. 1700)", El Arpa. Revista de la A.A.A.A, Nicanor Zabaleta, 2, 1991, p. 14. 
Droysen-Reber ${ }^{3}$. De acuerdo con Cristina Bordas, la invención de este instrumento de hileras cruzadas de cuerdas se le atribuye al violero Juan de Carrión, que hacia la segunda mitad del siglo XVI lo construyó en respuesta a las exigencias del creciente cromatismo en la práctica musical y al proceso paulatino de emancipación de la música instrumental ${ }^{4}$. La innovación de Carrión consistió en añadir a la fila de cuerdas diatónicas del arpa otra de "cuerdas específicas para los sostenidos y bemoles" dispuestas en grupos de 2-3-2-3, como en los instrumentos de tecla. Ambos órdenes se anudaban en la parte izquierda del clavijero, lo que producía su cruzamiento aproximadamente a $2 / 3$ de su longitud, quedando la parte más corta de las cuerdas hacia el cuello del arpa ${ }^{5}$. Este cruce fue lo que diferenció al arpa cromática española de la construida en Italia por la misma época, llamada arpa doppia, cuyos dos órdenes de cuerdas estaban dispuestos paralelamente ${ }^{6}$. La novedosa manera de realizar cromatismos posibilitó que a partir del siglo XVII el arpa de cuerdas cruzadas se introdujera como realizador del bajo continuo en las prácticas musicales de España y Portugal, donde se usó hasta la primera mitad del siglo XVIII En la actualidad se conservan en España y otros países europeos algunos

\footnotetext{
${ }^{3}$ N. Zabaleta: "The Harp in Spain from the XVI to XVIII Century", Harp News, 1, 8, 1953, pp. 2-9; El arpa en Espana de los siglos XVI al XVIII: antecedentes historicos: discurso del académico electo Nicanor Zabaleta Zala, leído en el acto de su recepción pública el día 24 de enero de 1988 y contestación del Duque de Alba, Madrid, Real Academia de Bellas Artes de San Fernando, 1988; H. J. Zingel: "Die kreuzsaitige. 'Arpa de dos órdenes"', Die Musikforschung, 7, 3, 1954, pp. 335-336; Harfenspiel im Barockzeitalter, Ratisbona, G. Bosse, 1974; S. Kastner: "Harfe un Harfner in der Iberischen Musik des 17. Jahrhunderts", Natalicia musicologica Knud Jeppesen septuagenario collegis oblata, Bjørn Hjelmborg y Søren Sørenson (eds.), Copenhague, Hansen, 1962, pp. 165-172; R. Stevenson: Christmas Music from Baroque Mexico, Berkeley, University of California Press, 1974, pp. 22-23; R. Pérez Arroyo: "El arpa de dos órdenes en España", Revista de Musicología, 2, 1979, pp. 89-107; J. López-Calo: Historia de la música española, 3, siglo XVII, Madrid, Alianza Editorial, 1983, pp. 59-64; C. Bordas: "The Double Harp in Spain from the $16^{\text {th }}$ to the 18 th $^{\text {th }}$ Centuries", Early Music, 15, 2, mayo de 1987, pp. 148-163; id.: "Origen y evolución del arpa de dos órdenes", Nassarre, V, 2, 1989, pp. 85 y 117; id.: "Die Spanische 'arpa de dos órdenes"', Historische Harfen-Historical Harps. Beiträge zur Theorie und Praxis Historischer Harfen-Theoretical and Practical Aspects of Historical Harps, Heidrun Rosenzweig (ed.), Basilea, Musik-Akademie der Stadt Basel, 1991, pp. 24-42; id.: "Harp Builders in Madrid (1578-1700)", Proceedings of the International Historical Harp Symposium, Utrecht 1992, Jan Hendrikus van Schaik (ed.), Ultrecht, STIMU, 1994, pp. 89-98; id.: "Arpa. I. España”, Diccionario de la Música Española e Hispanoamericana, vol. 1, Emilio Casares Rodicio (dir.), Madrid, SGAE-ICCMU, 1999, vol. 1, pp. 705-716; E. Bermúdez: "The Arpa de dos órdenes in the Sammlung Alter Misikinstrumente Kunsthistoriches Museum Vienna”, The Historical Harp: Proceedings of the International Historical Harp Symposium Berlin 1994, A. Riesthuis (ed.), Bunnik, Stichting Pro Musica Neerlandica, 1998, pp. 37-41; D. Droysen-Reber: "Europäische Harfen von der Renaissance bis in das frühe 20. Jahrhundert im Überblick", Harfen des Berliner Musikinstrumenten-Museums, Berlín, Staatliches Institut für Musikforschung Preußischer Kulturbesitz, 1999, pp. 18-22.

${ }^{4}$ C. Bordas: "Arpa. I. España”, p. 708.

${ }^{5}$ Ibid., pp. 708 y 709.

${ }^{6}$ C. Bordas: "Origen y evolución...", pp. 87 y 91.

${ }^{7}$ C. Bordas: "Arpa. I. España”, p. 710; J. López-Calo: Historia de la música..., p. 59.

${ }^{8}$ Ibid., p. 713.
} 
ejemplares del instrumento de la segunda mitad del siglo XVII y principios del XVIII ${ }^{8}$, lo que ha permitido realizar estudios comparativos de sus características organológicas?.

\section{El arpa de dos órdenes en dos catedrales novohispanas}

En el caso de la Nueva España, si bien se ha documentado ampliamente el uso del arpa -de manera genérica- en distintos ámbitos de la sociedad novohispana, incluida su importante participación en las capillas de música de las catedrales entre el segundo tercio del siglo XVII y buena parte del siglo XVIII, hasta hace poco no se habían localizado fuentes escritas, iconográficas ${ }^{10} \mathrm{o}$ instrumentos históricos que permitieran probar de manera fehaciente que el modelo con dos órdenes de cuerdas hubiera sido utilizado de ese lado del Atlántico ${ }^{11}$. La localización reciente de algunos documentos del primer tercio del siglo XVIII constata su uso en la capilla musical de la catedral de Puebla de los Ángeles y revela que por esta misma época el cabildo catedralicio de Valladolid (nombre antiguo de la actual Morelia) procuró contratar a músicos que supieran tocarla.

La primera de estas referencias se encuentra en un acta capitular de la catedral angelopolitana fechada en octubre de $1725^{12}$, en la que se consigna el nombramiento de Juan Corchado (1686?-1732) ${ }^{13}$ como arpista

\footnotetext{
${ }^{9}$ C. Bordas: "The Double Harp in Spain ...", p. 160; E. Bermúdez: "The Arpa de dos órdenes in the...", pp. 37-41.

${ }^{10}$ En las 122 obras artísticas en las que aparece la imagen del arpa y han sido recopiladas en la base de datos de Iconografía musical novohispana de la UNAM, solamente se representan arpas diatónicas. Este proyecto, dirigido por Eugenia Roubina y en el cual participo, está en proceso.

${ }^{11}$ Acerca de la difusión de este modelo de instrumento, Cristina Bordas apuntaba de manera cauta que "parece ser que la española no se practicó fuera de la península íbérica [...]". C. Bordas: "Arpa. I. España", p. 709. Por otra parte, para el caso novohispano, no obstante la carencia documental que entonces había, el musicólogo Aurelio Tello conjeturaba que, por el cromatismo de las obras conservadas en los archivos catedralicios del virreinato, era muy posible que el arpa de dos órdenes de cuerdas sí hubiera sido utilizada en las catedrales novohispanas. Esta idea fue expresada durante el VII Coloquio de alumnos del Programa de Maestría y Doctorado en Música de la UNAM realizado el 11 de junio de 2013 en la sala Huehuecóyotl de la entonces Escuela Nacional de Música.

${ }^{12}$ La existencia de este importante documento y del testamento de este músico fueron dados a conocer por Galia Hernández, quien publicó algunos fragmentos de los mismos en un texto de temática más amplia. Sin embargo, en la reflexión que elabora sobre el posible uso de este modelo cromático de arpa en Puebla, no distingue entre el arpa doble italiana (arpa doppia) y la española de dos órdenes, no obstante cita el trabajo de Bordas en el cual la autora española expone claramente la diferencia entre ambos instrumentos y los ámbitos geográficos donde se difundió. G. Hernández: "La capilla de música en el entierro de un obispo: el efecto sonoro en una ciudad episcopal (siglo XVIII)", Rituales sonoros en una ciudad episcopal. Puebla, siglos XVI-XIX, Monserrat Galí (coord.), Puebla, CIESAS-BUAP, 2013, p. 268; C. Bordas: "Arpa. I. España”, p. 709.

${ }^{13}$ En las informaciones aportadas por tres testigos en 1720 en la Real Casa de la Contratación de Indias, dos de ellos sostienen que Juan Corchado contaba para entonces con veinticuatro años, mientras que el otro afirma -según anotó el escribano- que tenía treinta y cuatro. Sevilla, Archivo General de Indias (AGI), Contratación, 5470, n. 2, r. 116, ff. 3r-4r, 25-VII-1720. En el testamento de este músico
} 
en esta capilla musical, quien había servido como tenor y ministril de este instrumento en la catedral de Sevilla entre 1710 y $1720^{14}$."Eminente en el instrumento de arpa de dos órdenes" además de compositor y cantor, luego de haber sido examinado por algunos de los musicos de la capilla y [...] haber demostrado una gran destreza y habilidad nunca antes vista"15, se acordó admitirle con un salario de seiscientos pesos por ejercer este ministerio, enseñar a tocar su instrumento a dos infantes del colegio y por "componer los villancicos y letras [...] segun el estilo moderno" 16 . En la documentación hasta ahora consultada, con excepción de los arpistas que llegaron a ser maestros de capilla, no se ha hallado información de que algún otro ejecutante de este instrumento haya recibido un salario semejante. Por ejemplo, en 1710, al músico que precedió a Corchado se le asignó un sueldo de cien pesos y, en 1751 en Valladolid, a Roque Jacinto Sánchez de los Dolores se le otorgaron trescientos pesos, cien por músico y doscientos por arpista. En Guadalajara, Marcos Garzón "por servir en las plazas de corneta y arpa" tenía asignados, en 1721, doscientos pesos y, en 1723, al ingresar a la catedral de Oaxaca, Juan de Florentín recibió la misma

se consigna su nombre como Juan Esteban Corchado, quien murió el primero de marzo de 1732 y fue enterrado en la catedral poblana. Archivo General de Notarías del Estado de Puebla (AGNEP), notaría n. ${ }^{\circ}$ 3, caja 192, f. [1r], 1732.

${ }^{14} \mathrm{G}$. Hernández menciona que Corchado fue probablemente "infante de la catedral de Coria de 1714 a 1719". G. Hernández: "La capilla de música...", p. 268. Sin embargo, tanto Juan como su hermano Pedro Corchado, ambos tenores, entraron a servir a la catedral de Sevilla en 1710, donde Juan permaneció hasta 1720, cuando pidió licencia "para pasar en esta flota a las Indias". Rosa Isusi Fagoga: Sevilla y la música de Pedro Rabasa. Sonidos de la catedral y su contexto urbano en el s. XVIII, Sevilla, Consejería de Cultura y Deporte-Centro de Documentación Musical de Andalucía, 2012, pp. 154. Recuperado el 2 de septiembre de 2015, de http://www.centrodedocumentacionmusicaldeandalucia.es/ estaticos/publicaciones//sevilla-musica-pedro-rabassa/sevilla-y-la-musica-de-pedro-rabassa-primera-parte.pdf; Sevilla, Actas de Cabildo: L-90, f. 171v, 13-X-1710; L-91, f. 71v, 1-VI-1711; L-96, f. 215r, 24-VII-1720, http://www.centrodedocumentacionmusicaldeandalucia.es/ estaticos/publicaciones//musica-catedralsevilla-autos-capitulares-vol2/4.pdf; http://www.centrodedocumentacionmusicaldeandalucia.es/estaticos/publicaciones//musica-catedral-sevilla-autos-capitulares-vol2/5.pdf (última consulta: 2-9-2015).

${ }_{15}$ Quisiera agradecer al investigador angelopolitano Gustavo Mauleón el haberme facilitado las fotografías de este y otros documentos de la catedral angelopolitana que se encuentran en el Archivo del Venerable Cabildo de la Catedral de Puebla (AVCCP), que en la actualidad no es posible consultar in situ, y que cito en este trabajo. Puebla, AVCCP, Actas de Cabildo, L-24, f. 190v, 19-X-1725.

${ }_{16} \mathrm{Ibid}$. Testimonio del trabajo que Corchado realizó como compositor son las 13 obras que de él se conservan en los archivos de música de la catedral de Durango, del Colegio de Santa Rosa de Santa María de Valladolid, de la Colección Sánchez Garza y, según ha identificado Drew E. Davies, en el de la Basílica de Guadalupe. Miguel Bernal Jiménez: El archivo musical del Colegio de Santa Rosa de Santa María de Valladolid (Siglo XVIII), [Morelia], Sociedad Amigos de la Música-Ediciones de la Universidad Michoacana de San Nicolás, 1939, p. 41; Thomas Stanford: Catálogo de los acervos musicales de las catedrales metropolitanas de México y Puebla, de la Biblioteca Nacional de Antropología e Historia y otras colecciones menores, México, INAH, 2002, p. 397; Drew Edward Davies: Catálogo de la Colección de Música del Archivo Histórico de la Arquidiócesis de Durango, México, IIE-UNAM, 2013, pp. 264-268; Aurelio Tello, Nelson Hurtado, Omar Morales Abril y Bárbara Pérez Ruiz: Colección Sánchez Garza: catálogo y estudio documental, México, Cenidim, en prensa. Agradezco a Bárbara Pérez el haberme permitido acceder a algunas partes de este último texto. 
cantidad. Finalmente, en 1752, al ser nombrado arpista y contralto en la catedral de México, José Pardo de Lago obtuvo como salario doscientos pesos, lo que representaba una tercera parte de lo que un cuarto de siglo antes ganaba Corchado ${ }^{17}$. El sueldo inusualmente elevado que se le otorgó no se explica si sólo se toman en cuenta la alta valoración y demanda que en las catedrales, y en especial en Puebla, había en ese momento por el instrumento de dos órdenes. También hay que considerar, aparte de la trayectoria, méritos, conocimientos y habilidades de este músico como ministril y cantor, que se le pagaba por enseñar a los niños y por desempeñarse como compositor. Esta última tarea generalmente la realizaba el maestro de capilla.

Aparte del claro señalamiento respecto al tipo de arpa que Corchado ejecutaba, este acuerdo de cabildo también es importante porque, entre otros aspectos, revela que la capilla de música de la catedral de Puebla llevaba varios años sin contar con un ejecutante de arpa de dos órdenes ${ }^{18}$. De esto se desprende, por una parte, que Diego Florentín, el arpista que a falta de un músico más capacitado había ocupado dicha plaza de forma interina durante quince años - de 1710 a $1725-{ }^{19}$, tocaba un arpa de un solo orden de cuerdas y, por la otra, que Pedro Gregorio Rodríguez, quien se desempeñó como arpista durante la segunda mitad del siglo XVII, ejecutara muy probablemente el arpa de dos órdenes ${ }^{20}$. Es decir, en una catedral de primera importancia como la poblana se utilizaron modelos de uno y dos órdenes, debido a que hubo períodos en que no se contó con músicos que supieran ejecutar este último. Ello podría ser indicador de dificultades en la enseñanza y difusión de la técnica para ejecutar el instrumento de cuerdas cruzadas en esas tierras.

La predilección por el arpa de dos órdenes como instrumento de acompañamiento en el ámbito catedralicio novohispano es corroborada también por un par de documentos provenientes de Valladolid. En el primero de ellos, fechado el 8 de octubre de 1730, se proponía al deán de esta catedral que el bachiller Bartolomé de Mafra Vargas fuese examinado en el ministerio del arpa y, en caso de ser aprobado, sirviera al arpista Gregorio Álvarez acompañando en el instrumento "de dos ordenes a las funciones"

\footnotetext{
${ }^{17}$ Puebla, AVCCP, Actas de Cabildo, L-21, f. 395r, 24-XI-1711; Guadalajara, Archivo Histórico de la Arquidiócesis de Guadalajara (AHAG), Actas de Cabildo, L- 9, f. 7v, 28-V-1721; Oaxaca, Archivo Histórico de Arquidiócesis de Antequera Oaxaca (AHAAO), Actas de Cabildo, L- 4, 230v, 19-I-1723; México, Archivo del Cabildo Catedral Metropolitano de México (ACCMM), Actas de Cabildo, L-41, f. 193r, 5-XII-1752.

${ }^{18}$ Puebla, AVCCP, Actas de Cabildo, L-24, f. 191v, 23-X-1725.

19 Ibid., L- 21, f. 315v, 1-VII-1710; L-24, f. 205v, 11-XII-1725.

${ }^{20}$ De 1653 a 1667, en la capilla de música de la catedral de Puebla hubo dos arpistas, pues además de Gregorio Rodríguez se encontraba sirviendo Nicolás Griñón, quien también era cantor y violonero. Puebla, AVCCP, Actas de Cabildo, L-12, f. 486v, 20-XII-1652; L-13, f. 9v, 14-I-1653; L- 15, f. 329v, 11 II-1667; L-21, f. 38, 4-XII-1703.
} 
de la iglesia ${ }^{21}$. En un informe fechado dos días después, el cantor Juan de Mendoza dio cuenta del examen realizado por Mafra, en el que mencionaba que el aspirante ofrecía "una abilidad sin doctura ni conosimiento alguno de el Arte de la Musica", además de que había confesado no saber pulsar el arpa de dos órdenes que, según el examinador, era "mas a proposito para [ejercer] dicho Ministerio"22. Otro de los presentes en el examen del bachiller Mafra, el arpista Agustín Pedraza, coincidía con el dictamen anterior y apuntaba que el aspirante "en el tañer del harpa [...] solo ejecuta en lo que llaman lirico con rasonable aire y suelto de manos pero sin arte que es lo esensial para el qulto dibino" 23 . Por lo señalado en los informes de los examinadores, puede deducirse que los arpistas G. Álvarez y A. Pedraza sí pulsaban el arpa de dos órdenes, sin embargo no contamos con documentos que nos permitan ser concluyentes a este respecto.

A la información de estas fuentes que permiten constatar o conjeturar la presencia discontinua del arpa de dos órdenes en las capillas de música de estos recintos eclesiásticos, vienen a sumarse otras que no sólo confirman lo anterior, sino que además revelan que este modelo de arpa también fue construido en el virreinato de México, específicamente en la ciudad de Puebla. El 14 de mayo de 1726, seis meses después de la contratación de Juan Corchado, el cabildo catedralicio dio los primeros pasos para que tan dotado músico enseñase a tañer a los infantes de coro $^{24}$.Visto el mal estado en que se encontraban los instrumentos del Colegio, los integrantes del cabildo acordaron que con dinero de la fábrica espiritual se le comprara un arpa al "artífice" don Bernardo Rodríguez, organista y violero poblano ${ }^{25}$. Finalmente fueron tres las que se adquirieron ese año: "para el servicio de esta santa iglesia" un "harpa de dos hordenes" por la que se pagaron veinticinco pesos, más doce pesos por "un cajón en que se guarda" 26 ; para el colegio dos arpas más, por las que el mismo Rodríguez recibió nueve pesos ${ }^{27}$. Aunque el documento no especifica las características de estas últimas, por la diferencia de precios entre estas (cuatro pesos y medio cada una) y la de dos órdenes (veinticinco pesos), se puede inferir que las destinadas al colegio

${ }^{21}$ Morelia, Archivo Histórico de la Catedral de Morelia (AHCM), Actas de Cabildo, L- 18, ff. 374v y $375 \mathrm{r}, 8-\mathrm{X}-1730$.

${ }_{22}$ Morelia, AHCM, Papeles, Leg. 64, f. 42r, 10-X-1730.

${ }^{23} \mathrm{Ibid}$., f. 31r, 11-X-1730. Al parecer Mafra no fue recibido y dos meses después a Gregorio Álvarez le fueron otorgados "100 pesos como arpista". Morelia, AHCM, Actas de Cabildo, L-18, f. 379v, 1XII-1730.

${ }^{24}$ AVCCP, Actas de Cabildo, L-24, f. 190v, 19-X-1725.

25 Ibid., L-24, f. 258r, 14-V-1726. Sobre la actividad de Bernardo Rodríguez como organero y afinador de este instrumento véase Gustavo Mauleón: "El organero novohispano José Chacón y el órgano parroquial de la villa de Atlixco (Puebla, México)", Anuario Musical, 63, enero-diciembre 2008, p. 66.

${ }^{26}$ AVCCP, Fábrica Espiritual, 2a y 3a cuentas, exp. 10, 27-V-1626, f. 63v y 64r.

${ }_{27}$ Ibid., 6-VII-1626, f. 110r. 
posiblemente eran instrumentos chicos y de un solo orden de cuerdas, quizás más apropiadas para que los infantes comenzaran a ejercitarse y a aprender las primeras nociones para acompañar.

La documentación catedralicia que hace referencia a la construcción del arpa de dos órdenes reviste gran importancia, ya que ni en las ordenanzas de violeros de 1568 que normaban la actividad del gremio en la Ciudad de México y sus alrededores ${ }^{28}$, ni en las cartas de aprendizaje poblanas de 1590 y $1690^{29}$, se especifica cuál era el modelo de arpa que un violero debía saber construir. Es decir, que son los documentos hallados en la catedral de Puebla los que revelan que el arpa española de dos órdenes se construyó en el virreinato de México.

Si bien se tiene constancia del uso del arpa cromática en la catedral de Puebla y muy posiblemente en la de Valladolid, Michoacán, cabría preguntarse qué sucedió en el caso de la capilla catedralicia de México. En los documentos consultados hasta ahora no se han hallado referencias explícitas a este tipo de instrumento y, por lo general, estos no ofrecen información acerca de su morfología. Sin embargo, de manera excepcional, en un acta de cabildo de 1751 se indicaba que era necesario adquirir "una arpa grande con los bordones y cuerdas precisas para los acompañamientos" 30 . En ese momento el coro de la catedral no contaba más que con "una arpa y es la chica", por lo que no correspondía "al buque de esta santa iglesia" por carecer de la cantidad de cuerdas necesarias que permitieran realizar el acompañamiento de forma debida ${ }^{31}$. En los registros de los dos pagos hechos por la fabricación de este instrumento no se hace alusión a las características del arpa que se mandó a hacer y sólo se apunta que el precio pagado por ella fue de cuarenta y cuatro pesos y dos tomines ${ }^{32}$. Por la mención que hace este documento a "los bordones y cuerdas necesarias" y la elevada cantidad que se pagó, así como por la importancia musical de la capilla catedralicia de México y la época en que se mandó a hacer, bien pudo haberse tratado de un instrumento con dos órdenes de cuerdas.

\footnotetext{
${ }^{28}$ Francisco del Barrio Lorenzot: Ordenanzas de gremios en la Nueva España. Compendio de los tres tomos de la Compilación nueva de ordenanzas de la muy noble, insigne y muy leal Ciudad de México, México, Talleres Gráficos, 1920, pp. 80-86.

${ }^{29}$ Estas dos interesantes cartas de aprendizaje fueron encontradas y dadas a conocer por G. Mauleón y Arturo Córdoba. De la primera de ellas se ofrecen algunos fragmentos y la versión completa paleografiada de la segunda de ellas fue publicada íntegramente en A. Córdoba y G. Mauleón: "Violeros' y 'Guitarreros' en Puebla durante el siglo XVII: algunos testimonios documentales”, Historia de la música en Puebla, Elio Huesca Martínez (ed.), México, Conaculta, 2010, pp. 79-100.

${ }^{30}$ México, ACCMM, Actas de Cabildo, L-41, f. 9v, 30-VI-1751. Agradezco a Eugenia Roubina el haberme puesto sobre la pista de este documento, al cual también J. Marín hace referencia en su tesis doctoral. Javier Marín: Música y músicos entre dos mundos: la catedral de México y sus libros de polifonía (siglos XVI-XVIII), tesis doctoral, vol. I, Universidad de Granada, 2007, p. 203.

${ }^{31} \mathrm{Ibid}$.

32 México, ACCMM, Fábrica Espiritual, 6: f. 39v, 21-X-1752; f. 42r, 23-I-1753.
} 
Como se puede observar, las actas de cabildo hacen referencia al arpa de dos órdenes en períodos de crisis en los que se hacía difícil encontrar a ejecutantes de dicho instrumento. Cuando esto no sucedía, en general estos libros de acuerdos no ofrecen detalles en torno a las características organológicas de las arpas, limitándose a informar de aspectos relacionados con la administración de la capilla, como el ingreso, las asignaciones, reducciones e incrementos de salario, o los permisos y peticiones que los músicos dirigían al cabildo. Continuar las búsquedas en diferentes ramos de los archivos catedralicios mexicanos y de otros países latinoamericanos, puede aportar nueva información que permita ahondar en el estudio del tema que nos ocupa, y del cual ahora solo estamos en la posibilidad de ofrecer algunas noticias escasas y dispersas.

\section{Un arpa de dos órdenes en el Querétaro decimonónico}

Hasta donde se ha documentado, el arpa de dos órdenes no trascendió el ámbito de las catedrales durante el virreinato, sin embargo, gracias al testimonio dejado por el historiador Valentín F. Frías (1862-1926), sabemos que entre los años de 1840 y 1870 hubo en Querétaro un arpista popular que tocaba un arpa con dos hileras de cuerdas. Se llamaba Francisco Silva, pero entre los queretanos fue conocido como "don Pancho el arpero"y -precisa Frías- "era el único en su género", dado que el arpa que ejecutaba magistralmente era "de dos órdenes para poder tocar cuanto se le ocurría con sus respectivos sostenidos y bemoles" 33 . Este "filarmónico" era contratado para amenizar las fiestas y paseos de las familias queretanas "merced a su notable habilidad en el arpa" 34 . Su repertorio se componía de "varsovianas y otros bailes de la época tocados al son del arpa", los cuales alternaba con intervenciones cantadas "de sus geniales y humorosas composiciones", como El cieguito y La Almoneda, siendo esta última "una relación en mal forjados versos, en la cual criticaba una almoneda habida en casa de una persona conocida por su mezquindad"35. Una fuente literaria también atribuye a este músico el haber compuesto La tragedia de la Carambada y lo ubica como participante en un fandango de bandoleros tocando Las Margaritas y Los Queleles, piezas que en ese entonces "estaban de moda y Pancho el Arpero las tocaba con mucho

\footnotetext{
${ }^{33}$ Valentín F. Frías: Leyendas y tradiciones queretanas, Querétaro, Imprenta de la Escuela de Artes de Sr. San José, 1900, p. 48. La primera versión que encontré del texto "Don Pancho Arpero" apareció publicada, sin firma, en un suplemento de El Universal Gráfico en los años treinta. Agradezco a Antonio Moreno el haberme informado que dicho texto formaba parte de Leyendas y tradiciones queretanas. "Don Pancho Arpero", Magazín del Gráfico, supl. de El Universal Gráfico, 19-II-1933, p. 12.

${ }^{34}$ V. F. Frías, Leyendas y tradiciones..., p. 149.

35 Ibid., p. 151.
} 
sentimiento"36. Según esta misma fuente, en la cual no se hace referencia al modelo de instrumento empleado por el músico, el arpista "lo mismo improvisaba versos que tonadas para sus corridos (tragedias, como él decía)" ${ }^{37}$, lo que coincide con el relato histórico, el cual da cuenta de que Francisco Silva "era medio poeta y sabía acomodarles tonada a sus composiciones" 38 .

La mención explícita que hace Frías respecto al modelo de arpa ejecutado por este músico y al repertorio que interpretaba, es importante porque habla de la pervivencia en el ámbito popular de un instrumento que había desaparecido de las catedrales mexicanas durante la segunda mitad del siglo XVIII y que en España había dejado de utilizarse hacía casi un siglo ${ }^{39}$.

Quedan aún muchas incógnitas por resolver, como por ejemplo, si el arpa de dos órdenes fue utilizada por otros músicos decimonónicos más allá de la geografía queretana, la manera en que llegó a manos de Francisco Silva, o si se trataba de un instrumento construido en el siglo anterior o de factura contemporánea al músico.

En resumen, las fuentes estudiadas dejan fuera de duda que el arpa de dos órdenes trascendió el marco geográfico ibérico. Si bien es necesario seguir documentando la presencia de este instrumento en tierras novohispanas e indagar si las noticias de su utilización durante el siglo XIX en Querétaro corresponden a un hecho excepcional o a una práctica musical difundida en otras geografias, la información aquí ofrecida viene a probar que el arpa española de dos órdenes también se insertó en las prácticas musicales de la América española.

Recibido: 14-3-2016

Aceptado: 17-7-2016

\footnotetext{
${ }^{36}$ La novela Carambada. Realidad mexicana fue escrita en 1941 por Joel Verdeja Sousse. La información que aquí se consigna fue citada por Enrique Flores: "Coplas de malhechores: La Carambada", La Copla en México, A. González (ed.), México, Colmex, 2007, pp. 99 y 100.

${ }^{37}$ Ibid., p. 100.

38 V. Frías: Leyendas y tradiciones..., p. 149.

39 En general, el proceso de desaparición del arpa se dio antes en las capillas de música ibéricas que en sus pares americanas, aunque hubo excepciones como Pamplona, donde de acuerdo con Javier Marín, quien cita a Gembero Ustárroz, el arpa fue utilizada hasta el siglo XIX. C. Bordas: "Arpa. I. España", p. 714; J. Marín: Música y músicos entre dos mundos..., p. 203.
} 